\title{
ESTIMATION OF THE IMPROVED METHOD OF GRIDS AND APPLICATION FOR DYNAMIC SOLUTIONS
}

\author{
Aryassov, G.; BarashKova, T. \& GornostajeV, D.
}

Abstract: The purpose of this paper is to develop and generalize the improved method of grids (method of finite differences) described in (Kollats, 1969). For obtaining of interpolation polynomials, the matrixes and method of uncertain coefficients are used. The essential simplification of the calculation formulae is received. The question of accuracy of the obtained solution is examined. The numerical results are presented. The use of overlapping of interpolation intervals allows increasing an accuracy of the solution. The calculation results show that it is possible to adjust the accuracy of the solution either by changing the degree of the interpolation polynomial or with the help of overlapping of intervals.

Key words: matrix equations, method of grids, method of uncertain coefficients, interpolation polynomial, overlapping of intervals
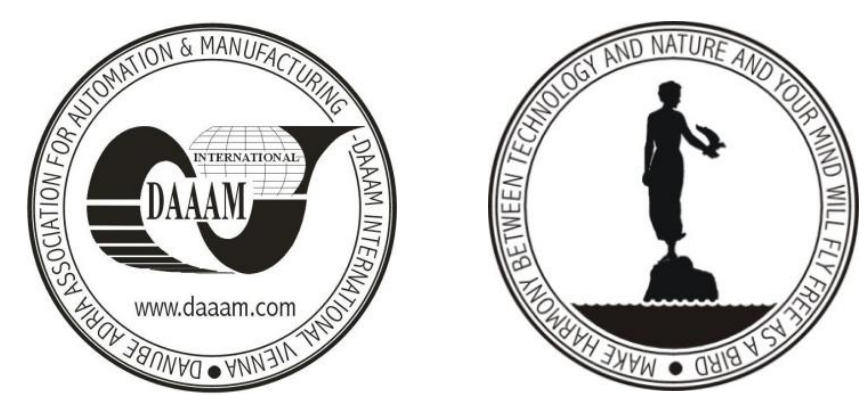

Authors' data: Associate Prof. Dr.techn. Aryassov, G[ennady]*; Dr. Sc. Barashkova, T[atjana]**, *Tallinn University of Technology, Ehitajate tee 5, 19086, Tallinn, Estonia, ** Virumaa College of TUT, Jarvekula tee 75, 30322, Kohtla-Jarve, Estonia, gennadi.arjassov@ttu.ee, tatjana.baraskova@ttu.ee

This Publication has to be referred as: Aryassov, G[ennady] \& Barashkova, T[atjana] (2013) Estimation of the Improved Method of Grids and Application for Dynamic Solutions, Chapter 31 in DAAAM International Scientific Book 2013, pp. 571-586, B. Katalinic \& Z. Tekic (Eds.), Published by DAAAM International, ISBN 978-3-901509-94-0, ISSN 1726-9687, Vienna, Austria

DOI: $10.2507 /$ daaam.scibook.2013.31 
Aryassov, G.; Barashkova, T. \& Gornostajev, D.: Estimation of the Improved Meth...

\section{Introduction}

In this paper we focus on partial differential equations in which of the parameters are not known exactly but affected by a certain amount of uncertainty, and hence described in terms of random variable (Marc, 2007). We shall confine ourselves to consider only the differential equation with zero regional conditions. In the case of general boundary conditions it is required to apply the matrixes, which are interpolated on Ermit. These matrixes turn out less elegant and more cumbersome, than the Vandermond matrixes, as both values of function and their derivative will be defined in this case. The method of uncertain coefficient can be used as for the traditional method of grids as for the "improved method of grids", which has been developed Kollats (Kollats, 1969) especially for solution of partial differential equations. The method of grids allows to reduce a task of continuous analysis to a problem of solution of system of the algebraic equations. The accuracy of the used interpolation polynomials is established by the well-known formulas from literature (nassar \& Eissa, 2003). This situation is quite common in the engineering practice. The theory of the method of finite differences is based on the theory of the approximation of functions, when values of them in discrete points are known. For this purpose, the interpolation polynomials obtained by the method of the uncertain coefficients are applied. Such approximation is possible to execute without resorting to the finite difference schemes (Jensen, 1972).

\section{Approximation by a Method of Uncertain Coefficients}

Let's consider the closed interval $[a, b]$, which is a part of wider interval $[A, B]$ (Fig.1). We set a task to approach the given function $\mathrm{y}=f(x)$. The task of dot approximation "forward" is solved by a method of uncertain coefficients (Miln, 1951). Arbitrary located an interval $[a, b]$ on an axis $x$ with length $l=b-a$, is divided on $n$ of equal parts of length $h=(b-a) / n$. Points of division or so-called nodes are designated in order of their following or increasing $\mathrm{x}_{0}, x_{1}, x_{2}, \ldots, x_{n}$ (Fig.1). Let's take into account, that $\Delta x=i \cdot h$; and $i=0,1,2, \ldots, n$. To these values $x_{i}$ correspond to the values of approximation function $\mathrm{y}_{0}, y_{1}, y_{2}, \ldots, y_{n}$.

It is required to find interpolation polynomial coefficients $P_{n}(x)$ of degree $\mathrm{n}$

$$
P_{n}(x)=a_{0}+a_{1} x+a_{2} x^{2}+\ldots+a_{n} x^{n},
$$

which graph passes consistently one time through all values $y_{i}, i=0,1,2, \ldots, n$ and only once. 


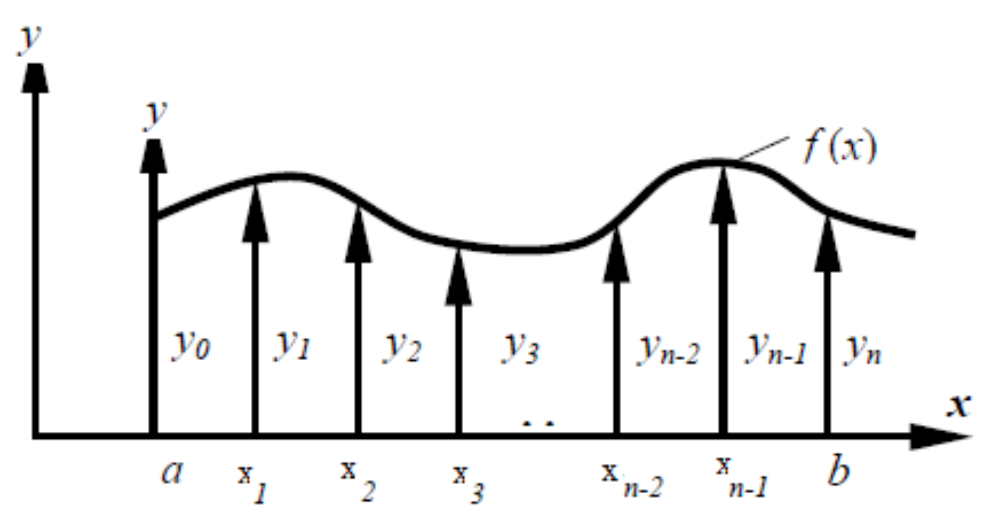

Fig. 1 The closed interval of $[a, b]$

For simplification of the task we make substitution of coordinates and use the dimensionless argument $\xi$, applying the following formulae

$$
x=a+\frac{b-a}{n} \xi ; 0 \leq \xi \leq n ; \xi=\frac{n(x-a)}{b-a}
$$

With the help of the formulas (2) it is possible to make transfer of a beginning of coordinates to a point $x=a$, that is to change scale on an axis $x$. After replacement of arguments $x$ in the interpolation polynomial (1) to dimensionless $\xi$ we receive

$$
P_{n}^{*}(\xi)=\alpha_{0}+\alpha_{1} \xi+\alpha_{2} \xi^{2}+\ldots+\alpha_{n} \xi^{n}
$$

The mutual transition from the polynomial (1) to the polynomial (3) and back is carried out with the help of the transformation formula (2). It is possible to write down the polynomial $P_{n}^{*}(\xi)$ in matrix symbolic as follows

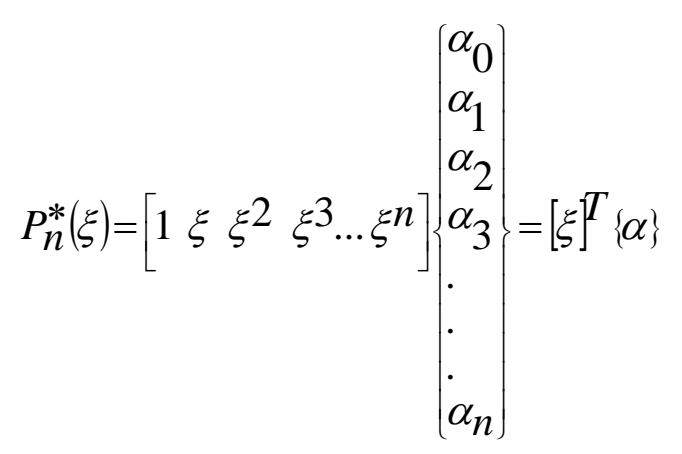

Substituting in the polynomial $P_{n}^{*}(\xi)$ the integer values of dimensionless argument $\xi$ and appropriate values of function $y$, the following system of the equations for calculation coefficients $\alpha$ is obtained: 
Aryassov, G.; Barashkova, T. \& Gornostajev, D.: Estimation of the Improved Meth...

$$
\begin{gathered}
P_{n}^{*}(0)=\alpha_{0}+0+0+\ldots+0=y_{0} \\
P_{n}^{*}(1)=\alpha_{0}+\alpha_{1}+\alpha_{2}+\ldots+\alpha_{n}=y_{1} \\
P_{n}^{*}(2)=\alpha_{0}+2 \alpha_{1}+4 \alpha_{2}+\ldots+2^{n} \alpha_{n}=y_{2} \\
P_{n}^{*}(3)=\alpha_{0}+3 \alpha_{1}+9 \alpha_{2}+\ldots+3^{n} \alpha_{n}=y_{3} \\
\quad \ldots . . . \\
P_{n}^{*}(n)=\alpha_{0}+n \alpha_{1}+n^{2} \alpha_{2}+\ldots+n^{n} \alpha_{n}=y_{n}
\end{gathered}
$$

This can be written down in a matrix form

$$
\left[W_{n}\right] \cdot\{\alpha\}=\{y\}
$$

Where

$\left\lfloor W_{n}\right\rfloor$-Vander monde matrix, which elements is degrees of a natural line numbers.

$$
\left[W_{n}\right]=\left[\begin{array}{ccccc}
1 & 0 & 0 & \ldots & 0 \\
1 & 1 & 1 & \ldots & 1 \\
1 & 2 & 4 & \ldots & 2^{n} \\
1 & 3 & 9 & \ldots & 3^{n} \\
\ldots & \ldots & \ldots & \\
1 & n & n^{2} & \ldots & n^{n}
\end{array}\right]
$$

The Vander monde matrix is not particular; therefore finding the inverse matrix to it is not difficult task and does not require the large efforts (Korn et al.). Multiplying the equation (6) at the left on $[W]^{-1}$ the line of coefficients $\{\alpha\}$ is determined

$$
\{\alpha\}=[W]^{-1}\{y\}
$$

Substituting expression (8) in the formula (4) we can present interpolation polynomials in the matrix form

$$
P_{n}^{*}(\xi)=\{\xi\}^{T}\left[W_{n}\right]^{-1}\{y\}=\left\{1 \xi \xi^{2} \ldots \xi^{n}\right\}^{T}\left[W_{n}\right]^{-1}\{y\}
$$

Approximate a broken line consisting of straight lines $y=0$ and $y=-12(\xi-2)$, by the fourth degree polynomial (Fig.2). 


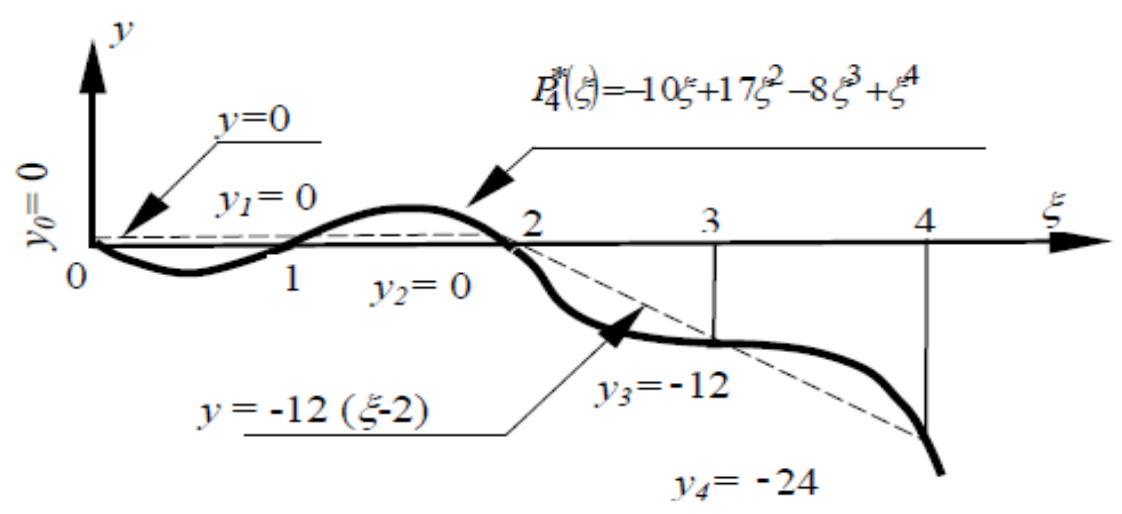

Fig. 2. Approximate a broken line

The calculation results of coefficients $\{\alpha\}$ by the formula (9) are given in a table form

\begin{tabular}{llllllll}
\multicolumn{7}{c}{$4 !\left[W_{n}\right]^{-1}\{y\}=\{\alpha\}$} \\
24 & 0 & 0 & 0 & 0 & 0 & 0 \\
-50 & 96 & -72 & 32 & -6 & 0 & -10 \\
35 & -104 & 114 & -56 & 11 & 0 & 17 \\
-10 & 36 & -48 & 28 & -6 & -12 & & -8 \\
$1-4$ & 6 & -4 & 1 & & -24 & & 1
\end{tabular}

As the matrix $\left[W_{n}\right]^{-1}$ is multiplied on 4 !, the coefficients $\{\alpha\}$ then should be divided on $4 !=24$.

In case of approximation of function on the extended interval $[A, B]$, which can consist of several intervals of a type $[a, b]$, are used the earlier received formulae. It is allowed, that intervals $[a, b]$ can overlap each other or be imposed against each other. On Fig. 3. one of such possible cases is shown.

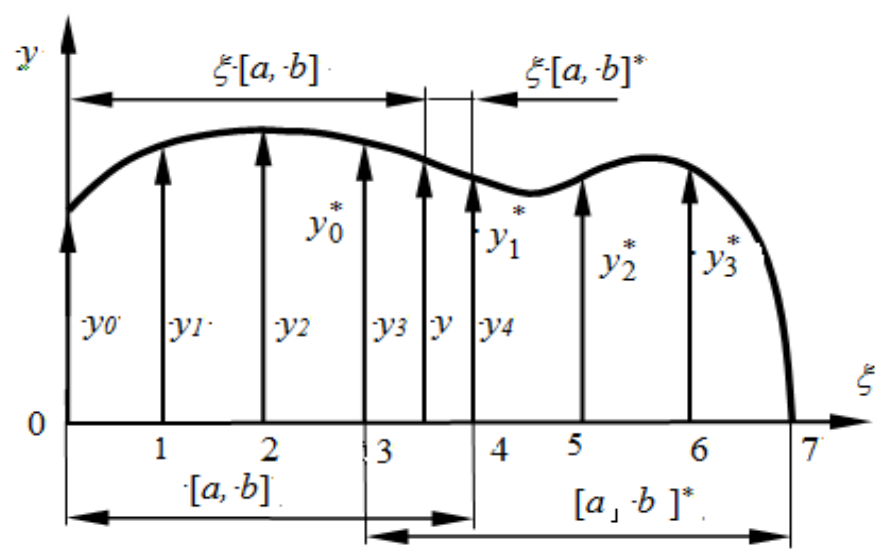

Fig. 3. The approximation function on the extended interval 
Aryassov, G.; Barashkova, T. \& Gornostajev, D.: Estimation of the Improved Meth...

The corresponding to an interval $[\mathrm{a}, \mathrm{b}]$ ordinates are denoted $\mathrm{y}_{0}, \mathrm{y}_{1} \ldots, \mathrm{y}_{\mathrm{n}}$. The corresponding to the next interval $[\mathrm{a}, \mathrm{b}]^{*}$ ordinates are denoted $y_{0}^{*}, y_{1}^{*}, \ldots, y_{n}^{*}$. The ordinates $y_{3}, y_{0}^{*}$ and $y_{4}, y_{1}^{*}$ coincide both on the location and on size. Intermediate ordinate in area, where there is "overlapping (Fig.3) is possible to receive by two ways:

1) Substituting in interpolation polynomials a value $\xi$ from the interval [a, b],

2) Substituting in interpolation polynomials value $\xi$ from the interval $[a, b]^{*}$.

In general case the given substitutions give various, but close results, this is explained by inexactitude of interpolation formulae. It is known, that the error of interpolation formulae is less in middle of an interval of interpolation and is great outside of it. In particular it is necessary to take into account this circumstance in calculation a derivative. Therefore it is expedient to use overlapping of intervals, especially, if the high order derivatives are to be calculated.

For preservation the accuracy of derivative calculations a higher order polynomial should be applied in comparison with polynomial which used for calculation of the function. The numbering of nodes is established in case of approximation of function in the extended interval [A, B]. In general case this numbering will differ from nodes numbering in the interval $[a, b]$.

It should be noticed, that the beginning of coordinates of interpolation polynomials can be changed arbitrarily, but to change the order of following of nodes and corresponding ordinates is not allowed in any event.

\section{Interpolation of Derivatives Function}

From the formula (3) follows, that for calculation a derivative of interpolation polynomial is sufficient to differentiate only matrix-line $\{\xi\}^{\mathrm{T}}$. Other multipliers of expression (9) are invariant to operation of differentiation.

Above-mentioned statement in identical degree concerns to operation of integration. For example, third derivative from the polynomial (9) will be defined as

$$
\frac{d^{3} P^{*}(\xi)}{d \xi^{3}}\left[000624 \cdot \xi 120 \cdot \xi^{2} \ldots n(n-1)(n-2) \xi^{(n-3)}\right]^{T}\left[W_{n}\right]^{-1}\{y\}
$$

It is possible with the help of the formula (11) to calculate derivative value in any point $\xi$ of the interval $[\mathrm{a}, \mathrm{b}]$. For example, at $\xi=1$ the third derivative value is equal

$$
\left.\frac{d^{3} P^{*}(\xi)}{d \xi^{3}}\right|_{\xi=1}=\left[\begin{array}{llllll}
0 & 0 & 0 & 24 & 120 & \ldots n(n-1)(n-2)
\end{array}\right]^{T}\left[W_{n}\right]^{-1}\{y\}
$$


It is important to know in the method of grids the derivative values in nodes of interpolation. These values are easy obtained from the formulae (12) if suppose, that $\xi$ accepts consistently the values as: $\xi=0,1,2, \ldots, n$. Then the line of $\mathrm{m}^{\text {th }}$ derivative from $[\xi]^{\mathrm{T}}$ becomes a square matrix. At $\mathrm{n}=4$ matrixes-columns or the vector-column second derivative from $P_{n}^{*}(\xi)$ will be

$$
P_{4}^{* \prime \prime}(\xi)=\frac{d^{2} P_{4}^{*}(\xi)}{d \xi^{2}}=\left[\begin{array}{lllcc}
0 & 0 & 2 & 0 & 0 \\
0 & 0 & 2 & 6 & 12 \\
0 & 0 & 2 & 12 & 48 \\
0 & 0 & 2 & 18 & 108 \\
0 & 0 & 2 & 24 & 192
\end{array}\right]\left[W_{4}\right]^{-1}\{y\}=[-] 4^{\prime \prime}\left[W_{4}\right]^{-1}\{y\}
$$

Where $[-]_{4}^{\prime \prime}$ is a matrix, which turns out as a result of the given operations. The lower index of the matrix specifies the polynomial order, and upper index - the derivative order.

For example, the expression (12) for third derivative will be written down as

$$
\frac{d^{3} P_{4}^{*}(\xi)}{d \xi^{3}}=[-]_{4}^{\prime \prime}\left[W_{4}\right]^{-1}\{y\}
$$

The foregoing formulae for differentiation of functions, which are given in discrete points, are generalization of the classical formulae of numerical differentiation. Their error can be appreciated similarly, as it is carried out in the classical methods.

Let's introduce a matrix $\left[O_{n}^{(m)}\right]$, which simultaneously carries out both operations of interpolation and differentiation of function given by a vector $\{y\}$

$$
\left[O_{n}^{(m)}\right]=[-]_{n}^{(m)}\left[W_{n}\right]^{-1}
$$

With the help of (15) the transition from the given differential equation to the appropriate system of the linear algebraic equations becomes simpler.

We shall confine ourselves to consider only the differential equation with zero regional conditions. In the case of general boundary conditions it is required to apply the matrixes, which are interpolated on Ermit. These matrixes turn out less elegant and more cumbersome, than the Vandermond matrixes, as both values of function and their derivative will be defined in this case.

So we have

$$
[g(\xi)] y^{\prime \prime}+[r(\xi)] y^{\prime}+\lambda[s(\xi)] y=\{f(\xi)\}
$$

With $y(0)=0$ and $y(n)=0$ 
Where $[g(\xi), \mid r(\xi)]$ and $[s(\xi)]$ are diagonal matrixes with the corresponding values of functions $g(\xi), r(\xi)$ and $s(\xi)$ in points or nodes of interpolation, $f(\xi)$ is free function in the right part in the same points or nodes.

Taking into account (14) and (15) the system of the linear algebraic equations in a matrix form will be

$$
\left.\left.[g(\xi)] \mid O_{n}^{\prime \prime}\right]\{y\}+[r(\xi)] \mid O_{n}^{\prime}\right]\{y\}+\lambda[s(\xi)]\{y\}=\{f(\xi)\}
$$

This system of equations (17) according to the distributive operation can be written down in more convenient form

Or

$$
\left.\left([g(\xi)] \mid O_{n}^{\prime \prime}\right]+[r(\xi)] \mid O_{n}^{\prime}\right]+\lambda[s(\xi))\{y\}=\{f(\xi)\}
$$

$$
[D]\{y\}=\{f(\xi)\}
$$

Where $[D]=[g(\xi)]\left[O_{n}^{\prime \prime}\right]+[r(\xi)]\left[O_{n}^{\prime}\right]+\lambda[s(\xi)]$ is a matrix operator of given differential equation. In case of constant coefficients the matrix operator becomes simpler. As all

$\mathrm{m}$ matrixes, included in equation (18) can be calculated beforehand, the inferring (composing) of the equations becomes considerably simpler. The solution of system of the algebraic equations (18) can be carried out with the help of a inverse matrix

$$
\{y\}=[D]^{-1}\{f(\xi)\}
$$

Such solution is especially convenient in case of a large number of the right parts $\{f(\xi)\}$. In this case the inverse matrix $[D]^{-1}$ will carry out a role of resolving equation.

\section{Numerical Results}

For an illustration we consider some elementary examples. Euler problem about a longitudinal bend. The differential equation of deflection curve in bend of beam (Fig.4) loaded by the longitudinal force $\mathrm{F}$ and using a dimensionless coordinate $\xi$, can be written as

$$
x=\frac{l}{4} \xi ; \xi=\frac{4 x}{l} ; \frac{d^{2} v}{d \xi^{2}}+\lambda v=0
$$

Where

$$
\lambda=\frac{F l^{2}}{16 E I}
$$


With the boundary conditions $v(0)=v(l)=0$.
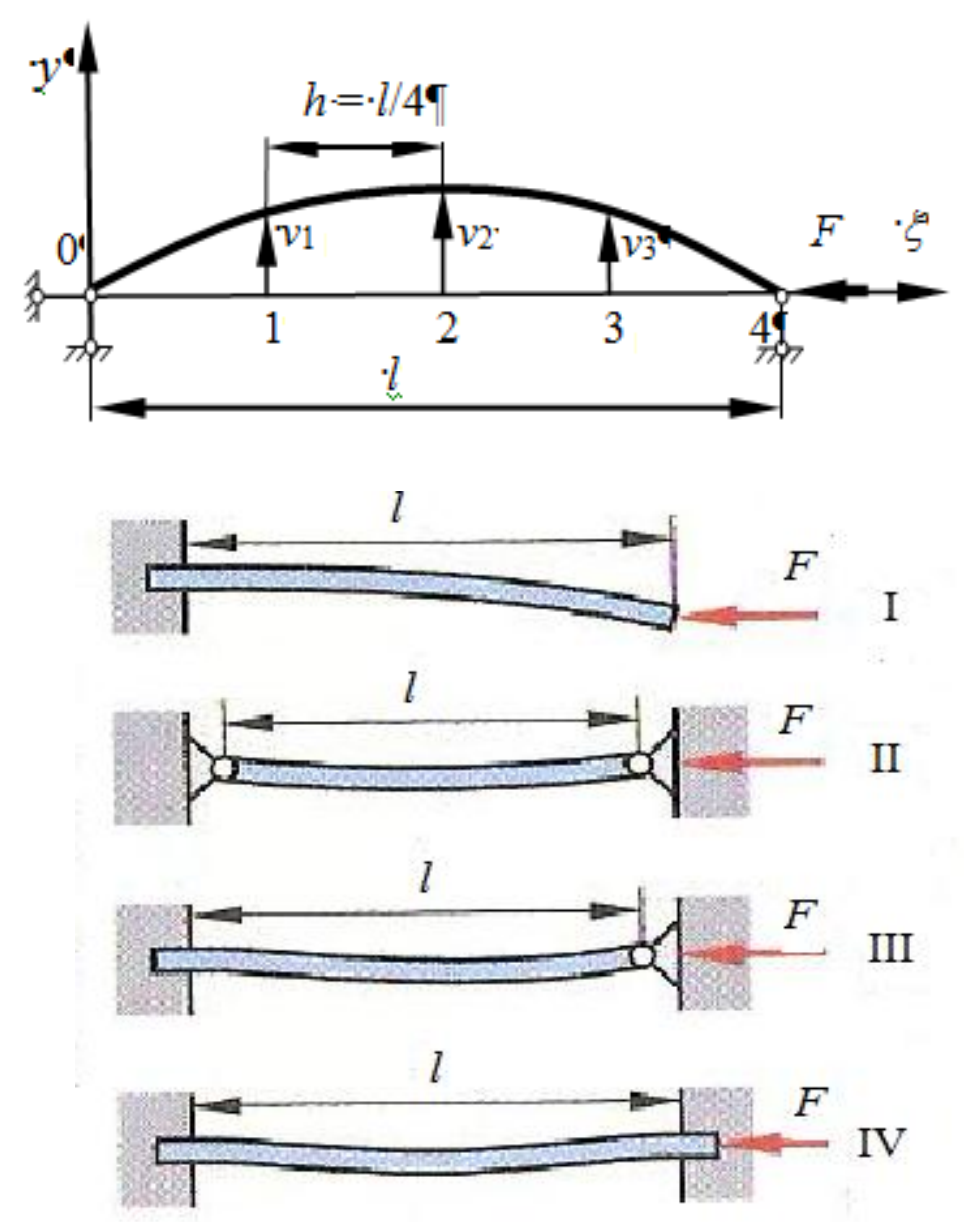

Fig. 4. Euler problem

The matrix operators $\left[O_{2}^{\prime \prime}\right]$ and $\left[O_{4}^{\prime \prime}\right]$ will be accordingly

$$
\left[O_{2}^{\prime \prime}\right]=[-]_{2}^{\prime \prime}\left[W_{2}\right]^{-1}=\left[\begin{array}{c}
002 \\
002 \\
002
\end{array}\right]\left[\begin{array}{ccc}
1 & 0 & 0 \\
-1,5 & 2 & -0,5 \\
0,5 & -1 & 0,5
\end{array}\right]=\left[\begin{array}{lll}
1 & -2 & 1 \\
1 & -2 & 1 \\
1 & -2 & 1
\end{array}\right]
$$

And

$$
\left[O_{4}^{\prime \prime}\right]=[-]_{4}^{\prime \prime}\left[W_{4}\right]^{-1}=\frac{1}{24}\left[\begin{array}{ccccc}
70 & -208 & 228 & -112 & 22 \\
22 & -40 & 12 & 8 & -2 \\
-2 & 32 & -60 & 32 & -2 \\
-2 & 8 & 12 & -40 & 22 \\
22 & -112 & 228 & -208 & 70
\end{array}\right]
$$

Using the expression (18) and operator $\left[O_{2}^{\prime \prime}\right](22)$ the characteristic system of the algebraic equations is obtained from equation (20), taking into account overlapping of intervals 
Aryassov, G.; Barashkova, T. \& Gornostajev, D.: Estimation of the Improved Meth...

$$
\begin{gathered}
2 v_{1}-v_{2}=\lambda v_{1} \\
-v_{1}+2 v_{2}-v_{3}=\lambda v_{2} \\
-v_{2}+2 v_{3}=\lambda v_{3},
\end{gathered}
$$

Which gives the critical force value for II (see fig.4)

$$
F_{k p}=\frac{9.38 E I}{l^{2}}
$$

With an error $5.2 \%$.

Applying the operator $\left[O_{4}^{\prime \prime}\right](23)$, according to the expression (18) in equation (20) without taking into account the overlapping of intervals, we receive the characteristic system of the equations with the same number of unknowns

$$
\begin{aligned}
& 40 v_{1}-12 v_{2}-8 v_{3}=\Theta v_{1} \\
& -32 v_{1}+60 v_{2}-32 v_{3}=\Theta v_{2} \quad \Theta=4 ! \lambda \\
& -8 v_{1}-12 v_{2}+40 v_{3}=\Theta v_{3}
\end{aligned}
$$

Which gives the value

$$
F_{k p}=\frac{9.395 E I}{l^{2}}
$$

With an error $5.0 \%$.

Increasing the division numbers or nodes (Fig.5), using operator $\left[O_{2}^{\prime \prime}\right]$, we receive

$$
F_{k p}=\frac{9.79 E I}{l^{2}}
$$

With an error $0.81 \%$.

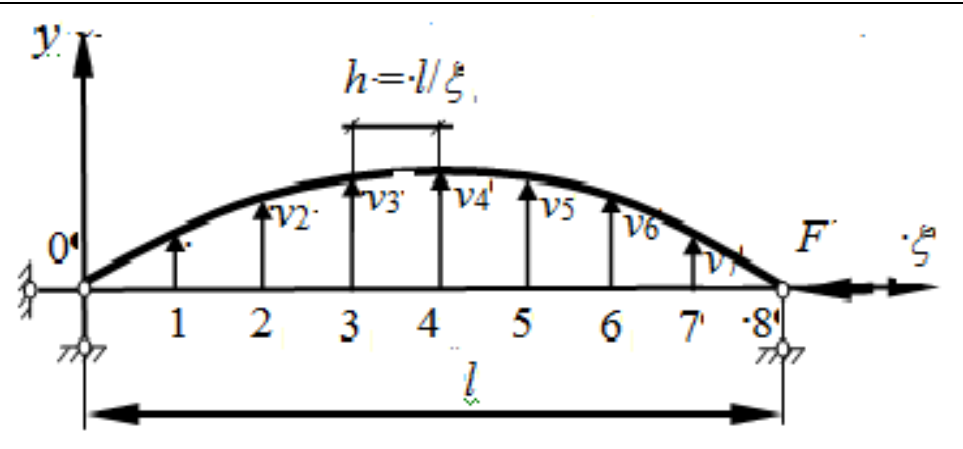

Fig. 5. Numerical results 
Applying operator $\left[\mathrm{O}_{4}^{\prime \prime}\right]$ in case of double number of nodes (Fig.5) and using the overlapping of intervals so, that a beginning of an interval $[a, b]$ is consistently combined with the nodes of the extended interval $[\mathrm{A}, \mathrm{B}]$ one after another $0,0,1,2$, $3,4,4$, the characteristic system of the equations is obtained, where

$$
\begin{array}{cc}
\lambda=\frac{F l^{2}}{64 E I} & \\
40 v_{1}-12 v_{2}-8 v_{3}+2 v_{4} & =4 ! \lambda v_{1} \\
-32 v_{1}+60 v_{2}-32 v_{3}+2 v_{4} & =4 ! \lambda v_{2} \\
2 v_{1}-32 v_{2}+60 v_{3}-32 v_{4}+2 v_{5} & =4 ! \lambda v_{3} \\
2 v_{2}-32 v_{3}+60 v_{4}-32 v_{5}+2 v_{6} & =4 ! \lambda v_{4} \\
2 v_{3}-32 v_{4}+60 v_{5}-32 v_{6}+2 v_{7} & =4 ! \lambda v_{5} \\
2 v_{4}-32 v_{5}+60 v_{6}-32 v_{7} & =4 ! \lambda v_{6} \\
2 v_{4}-8 v_{5}-12 v_{6}+40 v_{7} & =4 ! \lambda v_{7}
\end{array}
$$

The value of critical force will be $F_{k p}=\frac{9.87544 E I}{l^{2}}$ with an error $0.05 \%$.

\section{Two Dimensional Problem}

The above-mentioned technique for receiving ordinary derivative can be extended to calculation a partial derivative. Let's describe one possible variant. Let's accept additional notations and consider with the purpose of simplification a twodimensional problem only. We assume that a function $U$ of two variable is transformed using the dimensionless abscissa $\xi$ and ordinate $\eta$. The transformation is carried out with help of formula (2). The integer variables $\xi$ and $\eta$ form a square grid (Fig.6).

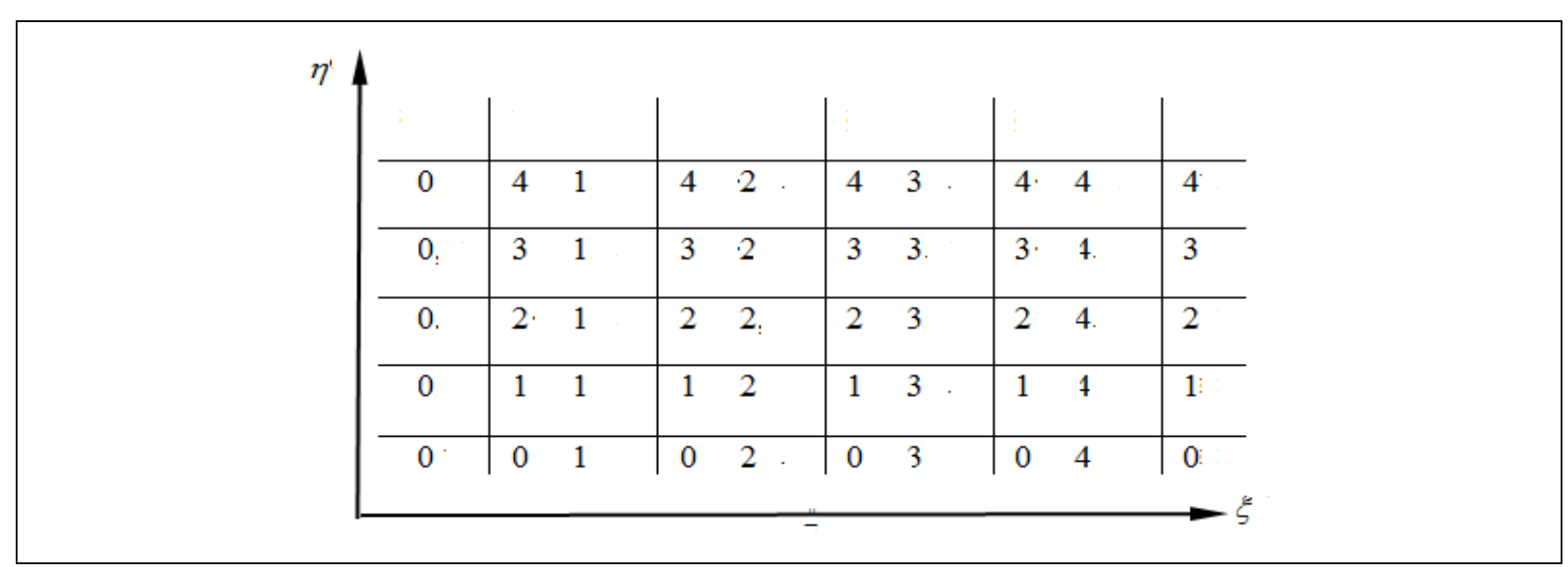

Fig. 6. Two dimensional problem 
Nodes of the grid are defined by two indexes. The first index corresponds to integer abscissa $\xi$, second - to ordinate $\eta$. It is expedient to join points laying on the same horizontal or vertical, that means having one and same abscissa or ordinate. Such association of points we shall name a vector-abscissa, with ordinate $\mathrm{k}$ and to denote $\left\{U_{j k}\right\}$ or vector-ordinate with abscissa i and to denote $\left\{U_{i \eta}\right\}$, where $\mathrm{k}=0,1,2$, $\ldots, \mathrm{r} ; r \leq n ; \mathrm{i}=0,1,2, \ldots, \mathrm{s} ; s \leq n$. Further in abbreviated form, accordingly "a vector $k$ "and "a vector $i$ ".

The symbol $\left[O_{n}^{(m)}\right\rfloor$ remains, but two indexes to it are added. They are written in brackets at the left and specify coordinates of a point or a vector-abscissa or a vectorordinate, along which the partial derivative are calculated. The upper index shows, according to the accepted notation, the order of the partial derivative.

Calculations of the partial derivative are carried out according to the abovementioned rules of numerical differentiation. Interpolation polynomial, appropriate to a vector $\left\{U_{i \eta}\right\}$, is differentiated on $\eta$. Let's consider an example, where the interpolation is carried out on five points, that is $n=4$. We assume that it is necessary to find the second partial derivative from the interpolation polynomial in the point $(2,1)$. Taking into account the expression (10) and the notation (13) yields

$$
\begin{aligned}
& (2,1)\left[O_{4}^{\partial^{2} / \partial \xi^{2}}\right] U={ }_{2,1}\left[O_{4}^{\prime \prime}\right]\left\{U_{\xi 1}\right\}=\left\{\begin{array}{lllll}
0 & 0 & 2 & 12 & 48
\end{array}\right\}^{T}[W]^{-1}\left\{U_{\xi 1}\right\}=
\end{aligned}
$$

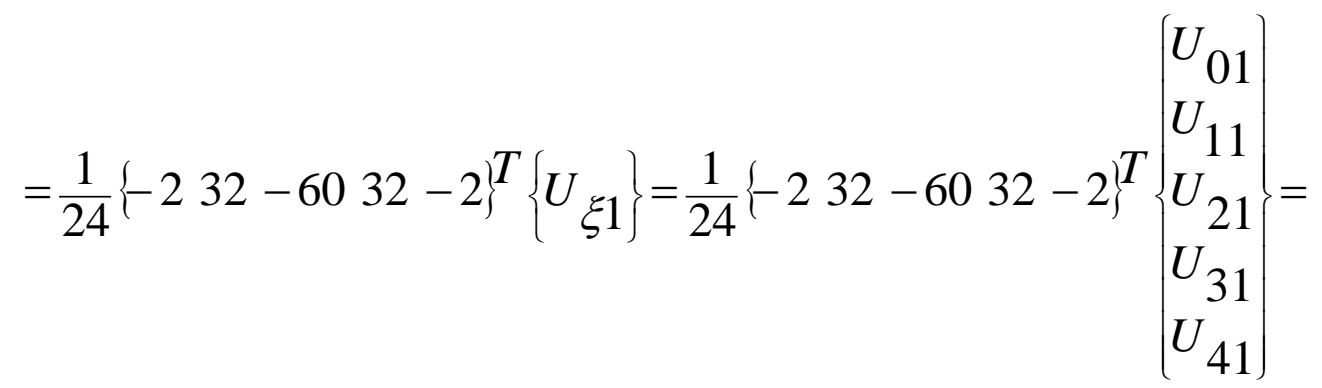

$$
\begin{aligned}
& \frac{1}{24}\left\{-2 U_{01}+32 U_{11}-60 U_{21}+32 U_{31}-2 U_{41}\right\} \text {. }
\end{aligned}
$$

To increase the accuracy of calculation of this derivative ${ }_{(4,2)}\left[O_{4}^{\partial^{2} / \partial \xi^{2}}\right] U$ in the point $(4,2)$ transfer the beginning of coordinates of the interval $[a, b]$ to the point $(4,2)$. It will correspond to differentiation of the interpolation polynomial, which is defined by a vector $\left\{U_{\xi 2}\right\}, \xi=2,3,4,5,6$.

The above-mentioned derivative can be calculated as 


$$
\begin{aligned}
& \left.(4,2)\left[O_{4}^{\partial^{2} / \partial \xi^{2}}\right] U=(4,2)\left[O_{4}^{\prime \prime}\right]\right\}\left\{U_{\xi 2}\right\}=\left\{\begin{array}{lllll}
0 & 0 & 2 & 12 & 48
\end{array}\right\}^{T}\left[W_{4}\right]^{-1}\left\{U_{\xi 2}\right\}=
\end{aligned}
$$

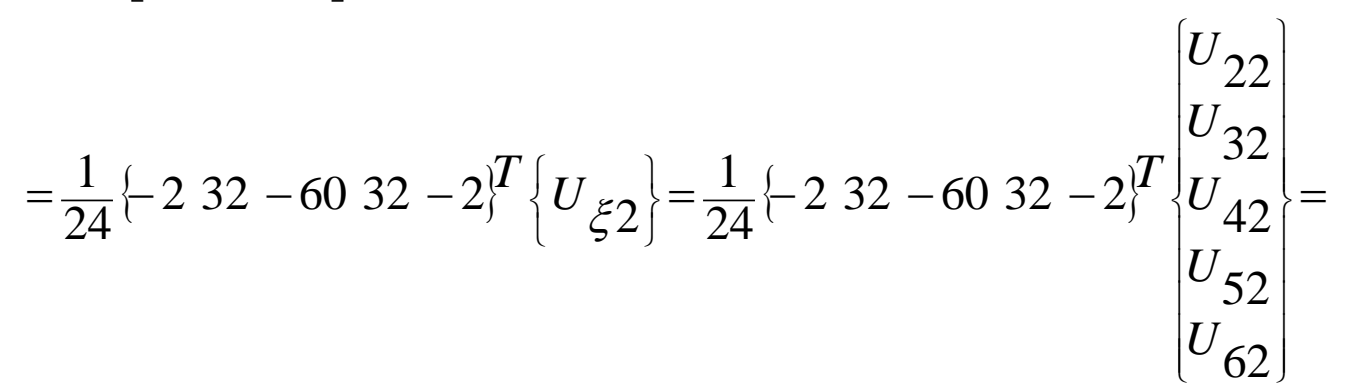

$$
\begin{aligned}
& =\frac{1}{24}\left\{-2 U_{22}+32 U_{32}-60 U_{42}+32 U_{52}-2 U_{62}\right\} \text {. }
\end{aligned}
$$

The calculation of partial derivative with respect of variable $\eta$ is made by a similar way.

Mixed partial derivative are calculated by the same rules, which are applied to interpolation polynomials along axes $\xi$ and $\eta$. In case of numerical differentiation on $\xi$ it is necessary to use so much vectors abscissa, how many points the used operator demands at numerical differentiation on $\eta$. For example, at $n=4$ five vectors are required. The result of calculation mixed derivative does not depend on the order of differentiation on $\xi$ and $\eta$. For increase of accuracy it is necessary to use overlapping of intervals.

Let's explain by an example. Let it is required to find a magnitude of fourth mixed derivative in a point $(2,1)$. This operation, taking into account the entered notation will be written down so

$$
{ }_{2,1}\left[O_{4}^{\partial^{4} / \partial \xi^{2} \partial \eta^{2}}\right] U={ }_{2,1}\left[O_{4}^{\partial^{2} / \partial \eta^{2}}\right]\left({ }_{2, \eta}\left[O_{4}^{\partial^{2} / \partial \xi^{2}}\right]\left\{U_{\xi \eta}\right\}\right)
$$

Here vector $(2, \eta)\left[O_{4}^{\partial^{2} / \partial \xi^{2}}\right]\left\{U_{\xi n}\right\}$ will be defined with the help of the formula

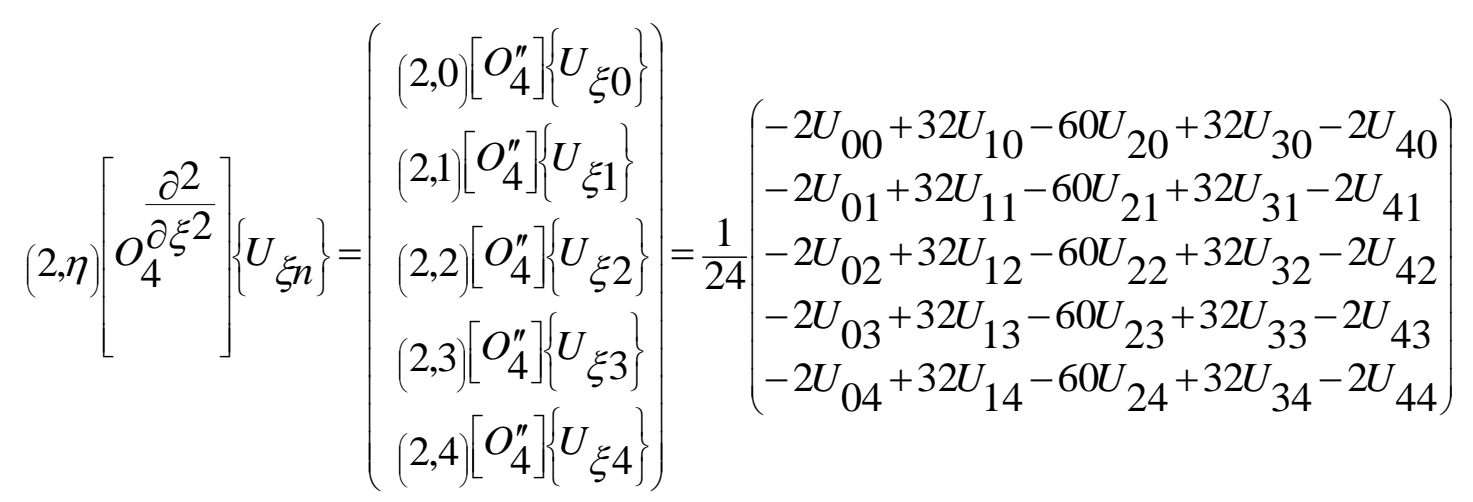


The calculation of the operator $(2,1)\left[O_{4}^{\partial^{2} / \partial \eta^{2}}\right]$ is reduced to finding of a matrix - line

$$
\begin{aligned}
& (2,1)\left[O_{4}^{\partial^{2} / \partial \eta^{2}}\right]=\frac{\partial^{2}}{\partial \eta^{2}}\left\{\begin{array}{lllll}
1 & \eta & \eta^{2} & \eta^{3} & \eta^{4}
\end{array}\right\}_{\eta=1}^{T}\left[W_{4}\right]^{-1}=\left\{\begin{array}{lllll}
0 & 0 & 2 & 6 & 12
\end{array}\right\}^{T} \\
& {\left[\begin{array}{ccccc}
24 & 0 & 0 & 0 & 0 \\
-50 & 96 & -72 & 32 & -6 \\
35 & -104 & -114 & 56 & 11 \\
-10 & 36 & -48 & 28 & -6 \\
1 & -4 & 6 & -4 & 1
\end{array}\right] \frac{1}{24}=\frac{1}{24}\left\{\begin{array}{lllll}
22 & -40 & 12 & 8 & -2
\end{array}\right\}^{T}}
\end{aligned}
$$

The required mixed derivative, expressed through values of the function $\left(U_{\xi, \eta}\right)$ in twenty-five points taking into account expressions (27) and (28), will have the following scalar expression

$$
(2,1)\left[O_{4}^{\frac{\partial^{4}}{\partial \eta^{2} \partial \xi^{2}}}\right] U=\frac{1}{576}\left(\begin{array}{c}
-44 U_{00}+704 U_{10}-1320 U_{20}+704 U_{30}-44 U_{40} \\
80 U_{01}-1280 U_{11}+2400 U_{21}-1280 U_{31}+80 U_{41} \\
-24 U_{02}+384 U_{12}-720 U_{22}+380 U_{32}-24 U_{42} \\
-16 U_{03}+256 U_{13}-480 U_{23}+256 U_{33}-16 U_{43} \\
4 U_{04}-64 U_{14}+120 U_{24}-64 U_{34}+4 U_{44}
\end{array}\right)
$$

If the interpolation will be carried out on three points (square parabola), that the second derivative operators in all nine points will be identical. In this case the fourth mixed derivative in the central point $(1,1)$ will be

$$
\begin{aligned}
& \left.(1,1)\left[O_{2}^{\frac{\partial^{4}}{\partial \eta^{2} \partial \xi^{2}}}\right] U=(1,1)\left[\frac{\frac{\partial^{2}}{\partial \eta^{2}}}{2}\right](1, \eta)\left[O_{2}^{\frac{\partial^{2}}{\partial \xi^{2}}}\right]\left\{U_{\xi \eta}\right\}\right)=\left\{1-21^{T}\left\{\begin{array}{l}
U_{00}-2 U_{10}+U_{20} \\
U_{01}-2 U_{11}+U_{21} \\
U_{02}-2 U_{12}+U_{22}
\end{array}\right\}=\right. \\
& =\left(U_{00}-2 U_{01}+U_{02}-2 U_{10}+4 U_{11}-2 U_{12}+U_{20}-2 U_{21}+U_{22}\right) .
\end{aligned}
$$

From general formula (26) we shall receive well-known result. Such approach for calculation derivative allows rather easily changing a step of a grid and number of nodes taken into account. In the case of change of the step it is necessary to resort to overlapping of intervals so that in any point the nodes of two next, adjacent intervals would coincide. In case of discrepancy of nodes it is necessary to calculate the interpolation polynomial values on square-law interpolation or more exact. 


\section{Conclusion}

The received formulae allowed to carry out the approximation of functions and their derivatives not resorting to differences as it is made in a classical method of grids (method of finite difference). The use of overlapping of interpolation intervals allows to increase an accuracy of the solution. The calculation results show that it is possible to adjust the accuracy of the solution either by changing the degree of the interpolation polynomial or with the help of overlapping of intervals. That is the main difference not only from usual, but also from the "improved" method of grids. The essential simplification of the calculation formulae is received, in particular case they are the L. Kollats' formulae. Their receiving is carried out with the help of matrix notable symbolic.

The received results can be applied to the solution of boundary problems of various classes, problem of eigenvalues etc. In particular, this is supposed to use the given approach for calculating the stress condition in threaded joints (Timoshenko, Arzyassov \& Strizhak, 1999, Haris \& McCrew, 1992). The last is especially urgent in vibrodiagnostic of structures, designs, machines, equipment, industrial and civil buildings and so on. The used matrix symbolic gives the convenient tool for realization of calculations with the help of computers. The possibility to omprove of methodoloqical basis of the Metrological assurance. Metrological assurance governs the quality of the products. Significance of metrological assurance is particularly obvious at the machine-building enterprises. Precision is the most important and decisive product-quality index. When solving the tasks of diagnostics the requirements to the precision of measuring devices are tighten by 1.5-1.6 times every year (Yosida, 1984, Aryassov \& Barashkova, 2009, Aryassov \& Petrishenko, 2009, Aryassov \& Petrishenko, 2008). In this connection measuring systems integrated into the net-communications of the enterprises become more and more necessary. At present there is disparity between possibilities of modern technological equipment and obstacles in the tasks of quality control both of technological process and manufactured products. The principle of production metrological assurance improvement is very urgent under existing conditions of manufacturing. It is necessary to create measuring information systems using the latest achievements of micro- and optoelectronics with computer processing of measuring results. Important feature of such systems is a possibility of measurements intellectualization. It is possible to improve present measuring complexes using modern technologies. Creation of a series of interference measuring systems with submicron and nanometric precision - is the guarantee of quality and safety.

\section{Acknowledgements}

This research work has been supported by Projekt SF0140113Bs08 (Mechatronic and Production Systems Proactivity and Behavioural Models) and by Estonian Archimedes Foundation (Project "Doctoral School of Energy and Geotechnology II", DAR 8130). 
Aryassov, G.; Barashkova, T. \& Gornostajev, D.: Estimation of the Improved Meth...

\section{References}

Marc P. Mignolet and Christian Soize, Stochastic Reduced Order Models for Uncertain Nonlinear Dynamical Systems. IMAC XXV, 2007, Orlando, Florida.

L. Kollats, Numerical methods of the decision of the differential equations. M., 1969. Nassar M.M., Eissa F.H, On the exponentiated Weibull distribution. Communications in Statistics. Theory and Methods, 2003

P. S. Jensen, Finite difference techniques for variable grids. Computer \& Structures, Vol. 2, Iss. 5, (Oct. 1972), pp. 17-29, doi:10.1016/0010-4485(72)90029-2 V.Miln, Numerical analysis. M., 1951

A. Korn, Theresa M. Korn. Mathematical Handbook edited by Granino, McCrewCompany New York, $831 \mathrm{p}$

S. Timoshenko strength of Materials Third edition 1996, Robert E Krieger Publishing Company, Malabar, Florida, USA

G.Aryassov, V.Strizhak To the Topic of Determination of Stress Concentration in Threaded Joints, 17th International Conference BEM/FEM99 Boundary\&Finite Elements Methods in Mechanics of Solids\&Structures, St. Petersburg, 1999

Shock and Vibration Handbook edited by Cyril H.Haris, McCrew- Hill Book Company, New York, 1992, 1312

K. Yosida. Operational Calculus. Springer, 1984. ISBN 0-387-96047-3

Aryassov, G. \& Barashkova, T. (2009). Mathematical methods for vibrations and their measurement. Materials, Methods \& Technologies, pp. 272 - 280, ISSN 13132539

Aryassov, G; Petritshenko, A. (2009). Study of Free Vibration of Ladder Frames Reiforced with Plate. J. Solid State Phenomena, pp. 368-373, ISSN 1662-9779 Aryassov, G; Petritshenko, A. (2008). Analysis of Stress Distribution in Roots of Bolt Threads. Pr. of the 19th International DAAM Symposium, pp. 0035-0036, ISSN 1726-967 